\title{
Pyridinolcarbamate; Bradykinin Antagonist, in Patients Suffering from Arteriosclerosis Obliterans and Thromboangiitis Obliterans (Buerger's Disease)
}

\author{
A Preliminary Report on the Treatment of Human \\ Atherosclerosis
}

Takio Shimamoto, M.D, and Toshiyuki Arsumi, M.D.

\begin{abstract}
Pyridinolcarbamate, a venous bradykinin antagonist, which has been shown to exert a curative as well as preventive effect on atheromatous lesions of cholesterol-fed rabbits, was tested in 2 patients suffering from obstructive diseases of lower extremities with intermittent claudication.

The first case was 33 year old man who had had cyanosis of his right foot and no pulsation of right dorsal pedal, posterior tibial and popliteal arteries. On pyridinolcarbamate the cyanosis disappeared on the 3rd day and the arterial pulsation reappeared on the $14 \mathrm{th}, 16 \mathrm{th}$ and 25 th day of the treatment respectively and the intermittent claudication improved concomitantly.

The second case was 57 year old man who had had no pulsation of his bilateral dorsal pedal, posterior tibial and popliteal arteries. On pyridinolcarbamate the pulsation of his right dorsal pedal artery reappeared on the 20th day of the medication with the concomitant improvement of intermittent claudication.

Pyridinolcarbamate seems promising in the treatment of human atherosclerosis.
\end{abstract}

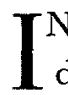

$\mathrm{N}$ this century there were three major lesions which were incurable showing dead spaces with the accumulation of dead substances such as gumma of syphilis, caseous materials of tuberculosis and atheromatous substances of atherosclerosis. Namely in these lesions there are factors rejecting the normal healing process by fibrosis.

Since the discovery of modern chemotherapeutic agents the former two are now curable and the atherosclerosis has been left for the solution of this problem.

In experimental study Shimamoto, Fujita and Numano $(1964)^{11}$ found that the administration of a venous bradykinin antagonist; pyridinolcarbamate $^{2), 3)}$ exhibits a curative effect on experimental atherosclerosis of cholesterol-

From the Institute for Cardiovascular Diseases and the Department of Medicine, Tokyo Medical and Dental University, Medical School, Yushima, Tokyo. 
fed rabbits. Namely the administration of this compound induced a rapid disappearance of edematous features in the atheromatous lesions and induced definitely a fibrous healing. The formation of atheroma and its fatty degeneration and necrosis were powerfully inhibited.

The authors ${ }^{3)}$ have attempted to see such an antiatherogenic effect of pyridinolcarbamate in man and many patients suffering from arteriosclerotic diseases of the brain and heart have been subjected to its clinical trial and a marked diminution or disappearance of anginal attacks and arrhythmias in patients suffering from coronary heart disease and a rapid restoration of some of neurological symptoms in patients suffering from cerebral arteriosclerotic disorders have been noted in over 1,000 cases during past 2 years period. However the direct evidence of the improvement of atheromatous lesion itself by pyridinolcarbamate is difficult to be evaluated in the arteries of these internal organs.

In order to see directly the effect on the arterial lesion the atherosclerosis of extremities seems suitable. Recently Wessler et al. (1960) ${ }^{4}$ have shown that the majority of cases of thromboangiitis obliterans (Buerger's disease) is nothing but atherosclerosis and the remaining few cases are the local thrombosis or embolism. Thus they negated the specificity of Buerger's disease.

The author could observe the dramatic effect of pyridinolcarbamate in one hospitalized patient suffering from thromboangiitis obliterans and in another hospitalized patient suffering from arteriosclerosis obliterans in the classical nomination and the results have been subjected to the present preliminary report.

\section{Materials and Methods}

Two patients; 33 and 57 year old men, hospitalized in our university hospital, were subjected to the present observation.

Pyridinolcarbamate was supplied by Banyu Co. Ltd. as Anginin-tablet containing $250 \mathrm{mg}$. in each tablet. This compound has been shown to antagonize competitively and reversibly the venoconstriction effect of bradykinin and its active homologues. Bradykinin induced venoconstrictive has been shown to cause local capillary dilation, congestion, exudation, thrombus formation and hemorrhage. All of these effect of bradykinin has been inhibited by this substance.

In this observation 2 tablets $(0.5 \mathrm{Gm}$.) of pyridinolcarbamate were given to the patients after each morning and evening meal, being $1 \mathrm{Gm}$. for the daily dose. Besides the routine clinical and laboratory examinations of our university hospital arteriography and plethysmography were utilized. The pulsation of each arteries was carefully recorded by electric recorder. The euglobulin lysis time was measured by Niewiarowski's method. ${ }^{5}$ The skin temperature of affected limbs was measured by electric thermometer of lío Co. Ltd. under the room temperature of $26-28^{\circ} \mathrm{C}$. 


\section{Case Reports}

Case 1. A 33 years old male had had occasionally pulsatile aches on his right ankle since 2 years especially in winter. Since the middle of last October he suffered from a tightening pain of his right calf after taking a walk of about 3 min. He could not walk further until after taking a rest of several min. and noted the typical rest pain of vascular insufficiency of his right calf. His intermittent claudication became steadily worse.

On December 10, he entered Tokyo University Hospital and he was told there his left ulnar artery and right dorsal pedal arteries were pulseless due to Buerger's disease. Angiography performed there revealed the stenosis of his right popliteal artery. He received hexaniacit and some other drugs for 7 months without any beneficial effect. His right foot became cool and since January of this year, his right foot exhibited a marked cyanosis and the cyanosis continued for 6 months without improvement. Finally he has been recommended the amputation of his right foot by surgeons of the hospital.

He had smoked 15 cigarettes a day, but there was no history of phlebitis, allergy, vasomotor instability or diabetes.

Immediately after the admission into our university hospital angiography revealed the closure of right popliteal artery and the most remarkable clinical findings were the marked cyanosis seen in his right foot and the perfect absence of the pulsation of right dorsal pedal, posterior tibial and popliteal arteries with intermittent claudication. The patient appeared somewhat older than his age of 33 . He was in distress from tightening sensation in the right calf. The distance to be able to walk without pain amounted $2 \mathrm{~min}$. The skin temperature of the right foot amounted $31.6^{\circ} \mathrm{C}$ which was lower than the left foot $\left(33.6^{\circ} \mathrm{C}\right)$ and the difference in temperature between the right and left foot amounted almost $2.0^{\circ} \mathrm{C}$ in the instep. The blood pressure was 112/68. The carotid, brachial, radial, and femoral pulses were palpable and good volume. The left popliteal, posterior tibial and dorsal pedal

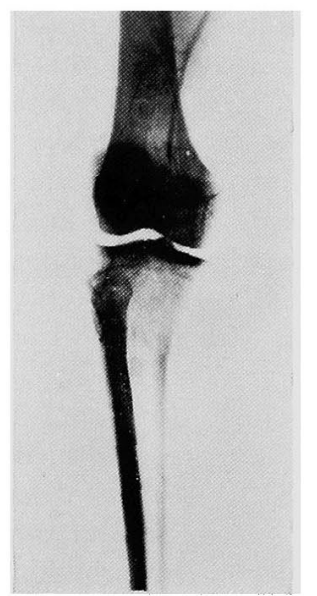

Fig. 1. The arteriogram of the right popliteal artery of the first case. Note: The closure of popliteal artery. 


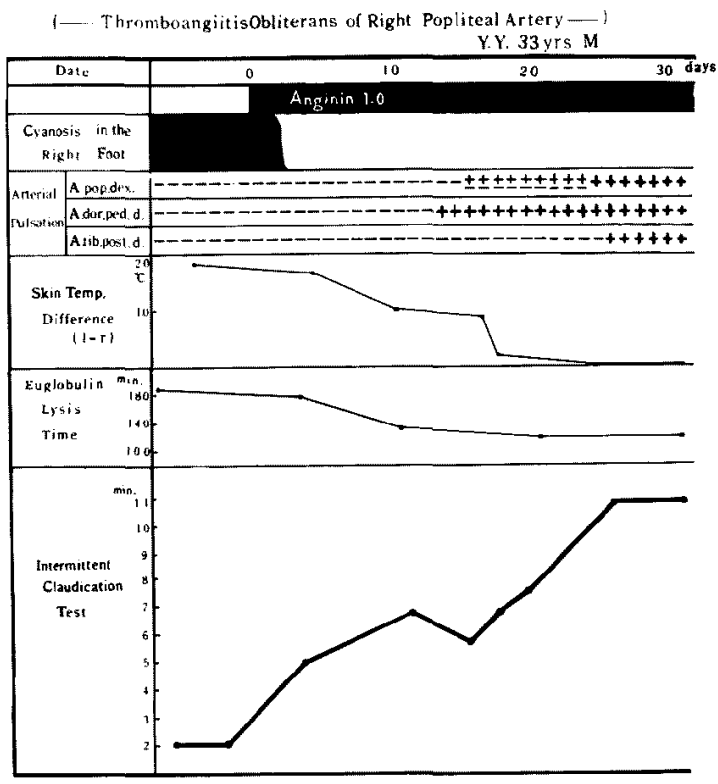

Fig. 2. Graphic representation of the course of Case 1 before and after pyridinolcarbamate treatment.

Note: The disappearance of cyanosis, the appearance of the pulsation'tof the right popliteal, dorsal pedal and posterior tibial artery, the gradual disappearance of the difference of skin temperature between the right and left foot, the gradual normalization of prolonged euglobulin lysis time and the increase in minutes of the walking without pain took place after the initiation of pyridinolcarbamate administration.

pulses were present and almost in the normal volume. The pulsation of the left ulnar artery was lacking.

The hemoglobin was $13.6 \mathrm{Gm}$./dl., the erythrocyte count $4,050,000$, the leucocyte count 6,450 , neutrophils 54 per cent, lymphocytes 35 per cent, monocytes 5 per cent, and eosinophils 6 per cent. The corrected sedimentation rate was 1.5. The serum cholesterol level was $116 \mathrm{mg}$. $/ \mathrm{dl}$. The serum protein was $7.0 \mathrm{Gm}$. with $3.7 \mathrm{Gm}$. of albumin and $3.3 \mathrm{Gm}$. of globulin. The blood sugar and blood urea nitrogen determinations and urinalysis were within normal limits. The L.E. cell preparations, ASLO, CRP and RA were negative. The serologic test for syphilis was negative. The chest X-ray study was normal. The electrocardiogram was normal.

One week of hospitalization with placebo treatment in our hospital gave him no symptomatic change.

Pyridinolcarbamate tablets in a dose of 4 tablets ( $1 \mathrm{Gm}$. ) a day divided into each 2 tablets were given on the 8th day of his hospitalization. As illustrated in Fig. 2 the cyanosis with the rest pain of calf disappeared dramatically on the 3rd day of the medication and the intermittent claudication showed concomitantly a definite improvement. The intermittent claudication became remarkably improved after 10 days of the medication, and on the 14th day of the treatment the pulsation of the right dorsal pedal artery became visible through the skin, and on 
the 15 th day the pulsation restored entirely, and 11 days after the appearance of the pulsation, i.e. on the 25 th day of the medication, the difference of the skin temperature between the right and left foot disappeared. On the 16th day of the medication the pulsation of right popliteal artery also reappeared and clearly noticed by the touch with fingers, and on the 25th day the pulsation gained enough an amplitude, and on the 26th day the pulsation of right posterior tibial artery also reappeared. His prolonged euglobulin lysis time exhibited also a gradual shortening as shown in Fig. 2. He was well under the treatment with pyridinolcarbamate for 3 months in the hospital. There was noted no untoward effect.

Case 2. A 57-year old man was admitted for treatment of intermittent claudication of the right foot of one year's duration. Since 4 months prior to admission the patient suffered from the same symptom on the left foot and the patient had noted the typical rest pains of vascular insufficiency on his right and also left calf. He could'nt walk over 3 min. without the pain of the calf. He had smoked 10 cigarettes a day for many years. There was no history of phlebitis, allergy, vasomotor instability, or diabetes. The family history was noncontributory.

The patient was a thin man who appeared not ill. The blood pressure was 152/88. Chest X-ray test revealed a slight enlargement of the left ventricle. ECG test revealed a slight ST and T depression in $V_{4}$ to $V_{6}$ and Master's two step test was positive. Eyeground was normal. Both feet were cool, however, there was no change in the skin. Both femoral arterial pulses were of adequate volume. The most important finding was the complete absence of the bilateral popliteal, dorsal pedal and posterior tibial pulses. The hemoglobin was $15.2 \mathrm{Gm} . / \mathrm{dl}$. The erythrocyte count 4,800,000. The leucocyte count was 4,900, 46 per cent neutrophils, 3 per cent eosinophils, 49 per cent lymphocytes, and 2 per cent monocytes. The urinalysis was normal and the corrected sedimentation rate was 2. The serum cholesterol level was $190 \mathrm{mg}$./dl. and the fasting blood sugar was normal. The serologic test for syphilis was negative. ASLO, GRP and RA were negative. Euglobulin lysis time was slightly prolonged being $173 \mathrm{~min}$. A left femoral arteriography revealed the obstruction of the left femoral artery.

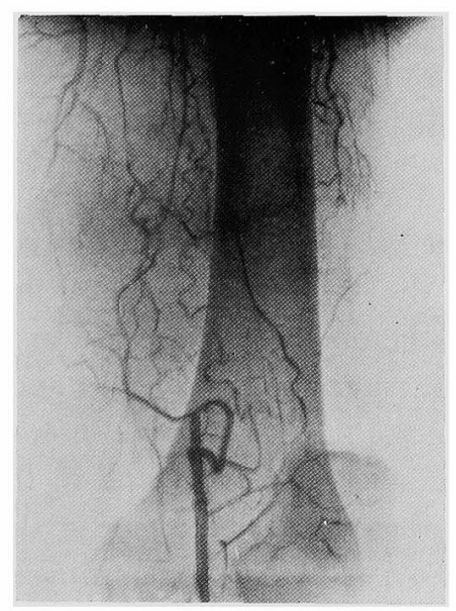

Fig. 3. The arteriogram of the left femoral artery. Note: The closure of the femoral artery. 


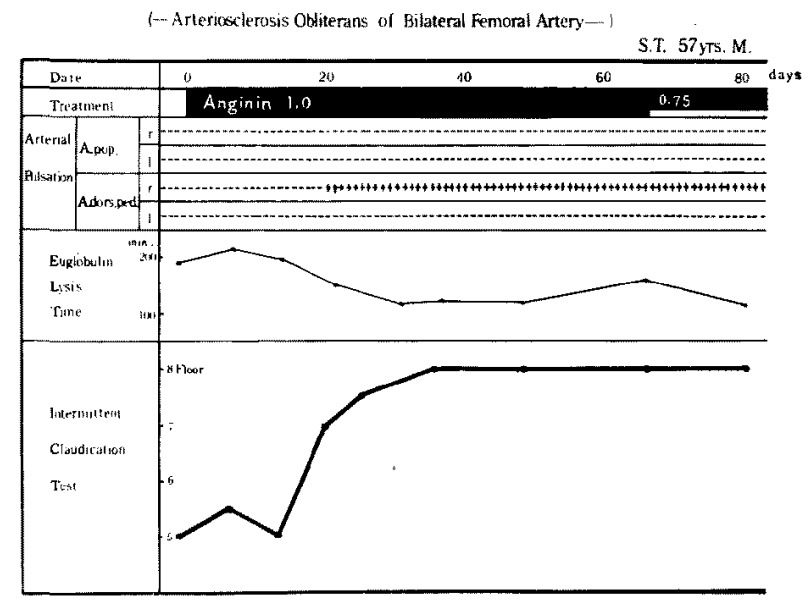

Fig. 4. Graphic representation of the course of Case 2 before and after pyridinolcarbamate treatment.

Note: The appearance of the right dorsal pedal artery, the normalization of prolonged cuglobulin lysis time and the increase in the number of floors on which the patient could step up without the pain of his legs, took place after the initiation of pyridinolcarbamate treatment.

After the treatment with pyridinolcarbamate for 2 weeks a slight improvement was noted in his intermittent claudication, and at the end of 3 rd week of the treatment a marked improvement in intermittent claudication was noted, and on the 20 th day a weak pulsation was clearly found in the right dorsal pedal artery. At the same time the intermittent claudication exhibited a remarkable improvement and the prolonged euglobulin lysis time shortened to nearly the normal value. After 2 months of hospitalization he was discharged and during 4 months period of the pyridinolcarbamate treatment he had no relapse. Moreover, in plethysmogram recorded on the 130th day of the treatment a definite shortening in crest time was found in bilateral second toes. There was noted no untoward effect in clinical and laboratory tests.

\section{Discussion}

In the first case there was lacking of an appreciable origin of embolism and thrombosis, so that the occlusive lesions of his extremities may have probably originated from atheromatous changes as in the case of Wessler et al.4)

The dramatic disappearance of the marked and long continued cyanosis seen on the 3rd day of the medication of pyridinolcarbamate in the first case shows the direct effectiveness of this compound in the occlusive lesions of affected artery. In the experimental investigation the edematous changes in the atheromatous lesions used to be rapidly improved on pyridinolcarbamate, so that the prompt disappearance of the cyanosis may have been induced by the rapid regression of the edematous changes of atheromatous lesions by 
pyridinolcarbamate. In patients suffering from anginal attack the disappearance of the attack took place often on the 3rd day of the medication of pyridinolcarbamate and such mode of effect of this compound may suggest the same situation. The appearance of the pulsation of affected arteries taken place on the 14th day of the medication may be originated from the further improvement of the atheromatous lesions.

The appearance of the pulsation of the dorsal pedal artery in the second case took place on the 20th day of the medication with the concomitant improvement of subjective and objective signs such as the shortening of prolonged euglobulin lysis time. The opening of collateral blood vessels might be possible for the improvement, however, the regression of the atheromatous lesions of affected artery seems probable, because in the course of the reappearance of the pulsation of the right dorsal pedal and popliteal artery in the first case the amplitude exhibited a gradual but relatively rapid increase during the following few days after the reappearance of its pulsation and reached to the almost normal pulsation. In addition the improvement of gait began concomitantly with the reappearance of the arterial pulsation in both cases and the improvement exhibited a relatively rapid progress. The further arteriographical analysis is obviously needed.

The duration of observation under pyridinolcarbamate treatment is still short in these cases amounting 3 months and 4 months respectively, however, no recurrence was observed during the whole period.

It has been well known that the traditional sympathectomy in the treatment of these morbid condition is so poor in its effect and even the opinion of not small number of investigators is against the operation today. Also no reliable compound has been known in the treatment.

The data obtained with pyridinolcarbamate seems highly promising and warrants further clinical trial in the treatment of atherosclerotic disorders.

\section{SUMMARY}

Pyridinolcarbamate; Anginin (B23) is a venous bradykinin antagonist, which antagonizes competitively and reversibly the venoconstrictive effect of bradykinin and its active homologues. This compound has also been shown to antagonize the congestion, exudation and hemorrhage induced by bradykinin and its active homologues and to remove the edematous change from experimental atheroma and to promote the normal fibrous healing of atheroma.

This compound has also exhibited clinically a beneficial effect in the atherosclerotic diseases of the brain and the heart, however, the evaluation of the effectiveness of this kind of drug in these morbid conditions had to depend 
upon rather the indirect evidences. In order to see directly the effect of pyridinolcarbamate on the arterial lesion the atherosclerosis of extremities seems much more suitable. The authors have first attempted this compound in the treatment of atherosclerotic occlusion in lower extremities of 2 hospitalized patients; 33 years old and 57 years old men.

The first patient aged 33 years suffered from a marked cyanosis of his right foot with a history of 6 months and the pulsations of right popliteal, posterior tibial and dorsal pedal arteries were completely absent and arteriography revealed the complete obstruction of popliteal artery immediately above the knee joint. Pyridinolcarbamate was given $1 \mathrm{Gm}$. daily and on the 3rd day of the medication the cyanosis disappeared dramatically and the intermittent claudication exhibited a slight improvement. On the 14th day of the medication the right dorsal pedal pulsation was recognized and on the 15th day the pulsation became visible through the skin and at the same time the pulsation of right popliteal artery began to be recognized. The volume of pulsation increased gradually but relatively rapidly and concomitantly with the remarkable improvement of intermittent claudication and on the 25th day of the treatment there was no differnce of skin tempcraturc between right and left foot and the pulsation exhibited almost normal volume in dorsal pedal artery. On the 26th day the pulsation of his right posterior tibial artery also reappeared. Under the medication of this compound no relapse and no untoward effects of the drug have been noted for 3 months period of the observation.

The second case aged 57 years suffered from intermittent claudication and the absence of the pulsation of bilateral popliteal, dorsal pedal and posterior tibial arteries and the arteriography revealed the occlusion of the middle part of the right femoral artery.

The treatment with pyridinolcarbamate for 2 weeks in a daily dose of one Gm. induced a slight improvement of intermittent claudication and on the end of third week a marked improvement was noted and at the same time the pulsation of his right dorsal pedal artery reappeared. His intermittent claudication exhibited a marked improvement concomitantly with the appearance of his dorsal pedal artery. A definite shortening in crest time in plethysmogram was noted in the bilateral second toes on the 130th day of the treatment. Thereafter his condition has been continuously good and he had no relapse and no untoward effects of the drug during the whole observation period of 5 months.

The effect of pyridinolcarbamate seems to originate from the improvement of atheromatous changes by the compound in these patients. 


\section{REFERENCES}

1. Shimamote, T., Fujita, T., and Numano, F.: Proc. of the Annual Meeting of Japan Pathological Association, 1965 (in press).

2. Shimamoto, T., Fujita, T., Kubota, M., Isokane, N., Yamazaki, H., and Numano, F. : Proc. of Asian Pacific Congress on Cardiology, 1964.

3. Shimamoto, T., Maezawa, H., Yamazaki, H., Atsumi, T., Fujita, T., Ishioka, T., and Sunaga, T. : Am. Heart J. (in press).

4. Wessler, S., Ming, S., Gurewich, V., and Freiman, D. G. : New Engl. J. Med. 262 : 1149, 1960.

5. Buckell, M. : J. Clin. Path. 11:403, 1958.

\section{ADDENDUM}

Since this manuscript was prepared, an additional series of 18 patients (male, age 33 to 62 yrs.) with arteriosclerosis obliterans have been subjected to pyridinolcarbamate treatment; the findings of this series are in agreement with the present report. The reappearance of dead arterial pulsation in the affected extremities took place on the 4th to 32nd day of the medication in 18 of all 24 affected arteries and the cyanosis observed in the affected feet of 6 cases disappeared on the 3rd to 5 th days of the medication and the ulcer observed in 4 cases responded dramatically to the treatment. 